\title{
Rheumatic diseases induced by drugs and environmental factors: the state-of-the-art - part one
}

\author{
Karolina Niklas ${ }^{1}$, Arkadiusz A. Niklas ${ }^{2}$, Dominik Majewski ${ }^{1}$, Mariusz Puszczewicz ${ }^{1}$ \\ ${ }^{1}$ Department of Rheumatology and Internal Diseases, Poznan University of Medical Science, Poland \\ ${ }^{2}$ Department of Hypertension, Angiology and Internal Medicine, Poznan University of Medical Science, Poland
}

\begin{abstract}
The majority of rheumatic diseases belong to the group of autoimmune diseases and are associated with autoantibody production. Their etiology is not fully understood. Certain medications and environmental factors may have an influence on the occurrence of rheumatic diseases. Establishing a cause-effect relationship between a certain factor and disease induction is not always simple. It is important to administer the drug continuously or monitor exposure to a given factor in the period preceding the onset of symptoms. The lack of previously diagnosed autoimmune disease, or finally the lack of symptoms within a few weeks/months after discontinuation of the drug/cessation of exposure, is also important. The most frequently mentioned rheumatic diseases caused by drugs and environmental factors include systemic lupus erythematosus, scleroderma, systemic vasculitis, polymyositis, dermatomyositis, and Sjögren's syndrome. The objective of this study is to summarize current knowledge on rheumatic diseases induced by drugs and environmental factors.
\end{abstract}

Key words: rheumatic diseases, drugs, environmental factors.

\section{Introduction}

Rheumatic diseases are mostly autoimmune diseases involving the production of antibodies against self-antigens. Their etiology and pathogenesis are not fully understood. A certain role is played by genetic and hormonal factors. In some cases, race seems to have an important role. An influence on the development of rheumatic diseases may also be exerted by medications and certain environmental factors. In order to prove that a certain medication causes a particular disease, several criteria must be taken into account: continued drug taking in the period preceding the onset of symptoms, the lack of early diagnosed autoimmune disease, and finally the cessation of symptoms within a few weeks/months after discontinuation of the drug.

Unfortunately, in practice, it is not always easy to determine a cause-effect relationship between a drug and the occurrence of symptoms of rheumatic disease, and one should remember that the establishment of the role of a drug in the occurrence of a disease is so important that its early discontinuation improves the prognosis. Autoimmune reaction to a substance cannot usually be predicted, as it depends on many factors, such as genetic susceptibility, the general health status of the patient taking into account concomitant diseases, and finally the interaction with other drugs, foods, and environmental factors [1]. The rheumatic diseases that are most often mentioned among those caused by drugs are systemic lupus erythematosus (SLE), systemic sclerosis, systemic vasculitis, polymyositis and dermatomyositis, and Sjögren's syndrome.

The objective of this study is to summarize current knowledge on rheumatic diseases induced by drugs and environmental factors. We performed a literature review from 2010 to 2015 using PubMed. In some reasonable cases we also included previous publications (especially those of historical value). Publications containing the key words "drug induced \& name of disease" and "environmental factors \& name of disease" were selected, 
and as the name of the disease in the first part of our study we used systemic lupus erythematosus and systemic sclerosis.

\section{Systemic lupus erythematosus induced by drugs}

The disease which is most often associated with taking drugs is SLE. The incidence of lupus in the United States is estimated at 15000 to 30000 newly diagnosed cases annually. About $10-15 \%$ of the cases are drug-induced lupus [2]. Currently, more than 90 drugs that can cause SLE have been described. The greatest risk, however, is associated with two: procainamide and hydralazine. According to literature data, among patients taking procainamide, lupus develops in $20 \%$ of cases during the first year of therapy. In the case of hydralazine, this percentage is 5-8\% [2]. Other drugs that may induce SLE are defined as of low or very low risk. The exception is quinidine, to which a moderate risk of lupus with a frequency of approximately $1 \%$ is assigned [1]. It is worth noting that even newer drugs may cause SLE. These include tumor necrosis factor (TNF) inhibitors, interferons, or agents used in cancer treatment $[3,4]$. Cases of SLE have been described after the use of cefepime (a fourth-generation cephalosporin), statins or ticlopidine $[5,6]$. Because hormonal factors are believed to play an important role in the etiology of SLE, estrogen contained in contraceptives must also be taken into consideration in drug-induced lupus. Bernier et al. confirm this hypothesis: the use of combined oral contraceptives increases the risk of SLE, especially in women who recently started oral contraception [7]. The situation is similar with hormonal replacement therapy: there is an increased risk of developing SLE in women who use such treatment for two or more years [8]. It is not clear if exogenous sex hormones may cause exacerbation of the disease in patients with diagnosis of SLE. It seems that hormonal contraception and replacement therapy are not contra-indicated in systemic lupus except patients with active SLE, severe disease activity at baseline, ischemic heart disease, prior history of thrombosis, positive anti-phospholipid antibodies or lupus anticoagulant $[8,9]$.

Drug-induced lupus is slightly different from the idiopathic variety. It is usually seen in the elderly, and there is no apparent gender predisposition, whereas for idiopathic lupus the male to female ratio is $1: 9$. Minocycline-induced lupus constitutes an exception - young individuals, mostly women, develop the disease more often. Drug-induced SLE affects Caucasian individuals six times more frequently than black individuals [2]. Genetic predisposition is also a factor: individuals who are so-called slow acetylators, or who have HLA-DR2, HLADR3 and HLA-DR4 haplotypes in their human leucocyte antigen (HLA) region, are more predisposed to the occurrence of drug-induced lupus [2].

Similar to in idiopathic lupus, the disease can manifest itself clinically as SLE, subacute cutaneous lupus erythematosus (SCLE) and chronic cutaneous lupus erythematosus (CCLE) [10]. The most common symptoms are general symptoms (such as fever, weakness, loss of appetite), arthritis, muscle pain, and inflammation of serous membranes. In general, in the course of drug-induced lupus, these symptoms are benign in comparison to idiopathic lupus; however, sometimes serious complications such as cardiac tamponade in the case of pericarditis are reported [11]. Kidney or central nervous system failure is rare $[1,2]$. Skin lesions occur in the form of characteristic facial erythema, but also discoid lupus erythematosus, and hypersensitivity to sunlight; moreover, hair loss and sores on the oral mucous membrane may also occur.

Laboratory tests on drug-induced lupus have confirmed the presence of antinuclear antibodies (ANA) and antibodies against single- and double-stranded DNA (ss-DNA and ds-DNA, respectively). High anti-dsDNA antibody titer is not specific for drug-induced lupus, and its presence may suggest the occurrence of idiopathic lupus. The exception is SLE induced by TNF inhibitors, for which these antibodies may be present in $90 \%$ of cases. The most characteristic for drug-induced lupus are anti-histone antibodies and, among them, antibodies of IgG class reacting with the native chromatin or ( $\mathrm{H} 2 \mathrm{~A}$ H2B)-DNA complex. It should also be added that many patients treated with drugs that may cause lupus have an elevated antibody titer against histones, although they do not exhibit clinical symptoms.

This titer gradually decreases after discontinuation of the drug that caused these alterations. In some cases of drug-induced lupus, particularly after application of minocycline and propylthiouracil, the presence of antibodies against neutrophil cytoplasm of perinuclear antineutrophil cytoplasmic antibody ( $p$-ANCA) has been identified. The presence of rheumatoid factor, anti-cardiolipin and anti-Smith (anti-Sm) antibodies has also been described. Other symptoms of drug-induced lupus may include an increased erythrocyte sedimentation rate (ESR), an elevated concentration of C-reactive protein (CRP), normochromic anemia, leucopenia, thrombocytopenia and hypocomplementemia [1, 2]. An interesting case in the light of laboratory tests was described by Stöllberger et al. [12]. An 89-year-old patient treated with dabigatran, which caused the occurrence of lupus, was diagnosed with acquired polyagglutination preventing blood group determination. It was only possible to 
obtain a correct result 66 days after drug discontinuation [12].

Treatment of drug-induced lupus is mainly based on discontinuation of the drug causing the disease. In resistant patients, glucocorticoids or hydroxychloroquine are sometimes used, and, in special cases, immunosuppressive drugs, e.g. azathioprine, cyclophosphamide or mycophenolate mofetil, are applied [2].

\section{Systemic lupus erythematosus versus environmental factors}

Environmental factors may have an impact on both the exacerbation of lupus erythematosus and the occurrence of new cases of the disease. The best-known environmental factor which exacerbates symptoms of lupus, particularly skin lesions, is ultraviolet (UV) radiation. Particularly sensitive to UV radiation are individuals for whom the presence of SS-A antibodies is reported. In these patients, this radiation induces apoptosis of keratinocytes, which results in the release of nuclear antigens, which increase the autoimmune response manifested by skin lesions [13].

Among the factors inducing the occurrence of SLE aromatic amines, hydrazine and silica dust have been considered. Especially in the latter case, studies confirm a significantly higher than expected incidence of lupus in men exposed to silica dust as an occupational risk factor [13]. Among other factors that may influence the development of the disease, tobacco is mentioned; however, the use of hair dyes, which was initially suggested as a strong etiological factor, has not been confirmed in subsequent studies [14]. Recently, estrogen-like substances such as organochlorines or organophosphates classified as pesticides have been taken into account as a possible SLE-inducing factor. Moreover, some plants consumed as food, such as yam or soybeans, contain high concentrations of phytoestrogens which can mimic the activity of estrogen.

Hence, there is speculation as to whether estrogens may contribute to an increased incidence of lupus, because it is known that in idiopathic lupus estrogens lead to exacerbation of the disease [13, 15]. Infections, especially viral infections, may appear to be an important causative agent. An association with the occurrence of lupus, as a consequence of infections, is suspected mainly in relation to Epstein-Barr virus (EBV), but also human endogenous retroviruses (HERV). HERV are an integral part of the human genome and, therefore, must be heritable. Mostly, they are inactive, but they may become a trigger factor in the autoimmune response via the phenomenon of molecular mimicry, because the similarity between amino acid sequences of HERV pro- tein products and lupus autoantigens has been demonstrated $[15,16]$.

One of the hypotheses of the pathogenesis of lupus caused by environmental factors concerns Toll-like receptors (TLRS). In spite of playing a role in the protection of the host, some studies suggest that, in certain conditions, TLRs may be responsible for autoimmune diseases [17].

\section{Systemic sclerosis induced by drugs}

Systemic sclerosis is a connective tissue disease. It affects women more often than men. It is mainly characterized by induration of the skin, but also fibrotic damage to internal organs and microcirculation disorders. Raynaud's phenomenon is an early symptom. Thereafter, gastrointestinal tract, lung or kidney failure often occurs. Serological markers are antibodies against topoisomerase I (anti-Scl-70). Both scleroderma and pseudoscleroderma can be induced by certain drugs. Bleomycin is the most frequently mentioned. It is a cytotoxic antibiotic and is widely used in cancer chemotherapy schemes [18].

Other pharmacological agents that may cause scleroderma include paclitaxel, pentazocine, cocaine, appetite suppressants and D-penicillamine. Paclitaxel is a cytotoxic alkaloid used in cancer chemotherapy. Pentazocine is an analgesic medication. Its chronic subcutaneous or intramuscular use can lead to disorders in microcirculation and ischemia, resulting in sores. Similarly, cocaine and appetite suppressants, such as diethylpropion hydrochloride, mazindol, amphetamine, dexamphetamine, phenmetrazine and methaqualone, have sympathomimetic activity and cause contraction of blood vessels.

Cocaine may also cause diffuse scleroderma-like alterations on the skin. However, appetite suppressant drugs are responsible for Raynaud's symptom, hand edema, sclerodactyly or difficulties in swallowing. D-penicillamine used in the treatment of several rheumatic diseases including systemic sclerosis has also been specified as a causative agent of scleroderma-like alterations, but these incidents have been described as rare [13]. Localized scleroderma (morphea), as a form of disease which affects only the skin and subcutaneous tissue, may constitute an effect of drug action. The association with this type of disease has been confirmed in relation to TNF inhibitors. In some patients who had previously been diagnosed with systemic sclerosis, anti-TNF therapy induced the occurrence of macrophage activation syndrome [5]. Morphea-like alterations have also been found in patients after treatment with balicat$\mathrm{ib}$ - a cathepsin $\mathrm{K}$ inhibitor, which is used in the treatment of osteoporosis [19]. 


\section{Systemic sclerosis versus environmental factors}

Scleroderma and scleroderma-like syndromes can also be induced by environmental factors. In contrast to idiopathic scleroderma, no evidence has been observed of a higher incidence rate in women. Most commonly with the occurrence of scleroderma, an association between exposure and silica dust is described. The mechanism of this phenomenon is not completely understood. It is suggested that the process is initiated by phagocytosis of silicon crystals through macrophages from the pulmonary alveolus. It causes activation of macrophages, but also endothelial cells, which leads to the release of cytokines responsible for symptoms appropriate for scleroderma. Clinically, the course of the disease resembles idiopathic scleroderma, although it may more often lead to pulmonary fibrosis. In most patients, anti-Scl-70 antibodies are also present. The results of the studies show that men are more likely to develop scleroderma after exposure to silica dust. Therefore, the need to wear protective masks in places exposed to silica dust is emphasized, and those working in such conditions should undergo detailed examination of the respiratory system $[13,20]$.

The question of whether silicones, especially those synthetic organosilicon polymers used in medicine as implants, can also induce systemic sclerosis has become an individual problem. The first reports of such a possibility appeared in the 1960s: cases were found of scleroderma-like syndromes of diffuse skin lesions, sclerodactyly, Raynaud's phenomenon and finger sores in women who had previously had mammoplasty using silicone injections. In addition, in these patients the presence of rheumatoid factor, antinuclear antibodies or antibodies against DNA was identified.

In the 1990s, regular reports on associations between cosmetic breast correction using silicone implants and the development of connective tissue diseases began to appear; however, several large epidemiological studies indicated only a small or no association between these two issues [11]. However, so far no clear answer regarding the safety of silicone implants has been obtained, and this issue requires further study, especially since we can still find new disturbing case studies (e.g Al Aranji et al. regarding a 52-year-old woman, for whom fracture of a silicone breast implant was reported, which consequently led to the rapid development of scleroderma-like skin lesions with subsequent scleroderma renal crisis) [21].

Apart from silicon compounds, there is a whole range of other substances that may be associated with the occurrence of scleroderma symptoms. This includes vinyl chloride. In the mid-1960s, there were the first reports of individuals working on the polymerization of vinyl chloride, who experienced finger paresthesia, cold sensation, Raynaud's symptom, clubbed fingers, osteolytic lesions in distal phalanges, edema and hardening of the skin of the fingers, hands and forearms, and pulmonary changes. The risk of the occurrence of symptoms increased with exposure frequency [22]. In laboratory studies, there were found increased concentrations of IgG, cryoglobulinemia, complement system activation and ANA in low titers [23]. After cessation of exposure, an improvement was most often observed, and a basic recommendation for prevention is to control the duration of exposure to vinyl chloride [13].

Other factors causing scleroderma include organic solvents. They include toluene, benzene, xylene, mineral spirits, perchloroethylene and trichlorethylene. Exposure to these substances results in the appearance of Raynaud's symptom, weakness, muscle pain, skin edema and sometimes neuropathies [24-26].

Alterations in the characteristics of skin sclerosis and muscle weakness are also caused by epoxy resins. Even short-term inhalation of their vapors may lead to alterations. Improvement is observed after cessation of the exposure [27].

It appears that not only chemical but also physical agents should be taken into account in the induction of scleroderma. Attempts have been made to confirm the relationship between exposure to vibrations and the occurrence of the disease. The test results of studies are not straightforward and require further follow-up [28].

\section{Toxic oil syndrome and eosinophilia- myalgia syndrome}

Toxic oil syndrome and eosinophilia-myalgia syndrome are two disease entities with similar courses, for which the etiology is associated with the consumption of contaminated food. Both of these entities were described in the 1980s and were epidemic. The epidemic of toxic oil syndrome began in 1981 in Spain. The cause was the consumption of rapeseed oil contaminated with aniline, which was intended for use in industry, but was illegally sold for human consumption. In turn, eosinophilia-myalgia syndrome appeared in 1989 for the first time in the United States. Its presence is associated with the consumption of L-tryptophan, which was a popular nutritional supplement at that time. Probably, contamination of L-tryptophan by 3-(phenylamino)-alanine led to the occurrence of the epidemic.

Clinical symptoms in both cases were similar: muscle pain (myalgia), arthralgia, sicca syndrome, scleroderma-like skin lesions with contractures of joints, pulmo- 
nary hypertension, and neuropathies. Mortality in both entities was also similar - approximately 2.5\% [29]. In laboratory tests, peripheral eosinophilia was characteristic for both diseases. In the treatment, glucocorticoids were used, which resulted in a reduction in the number of eosinophils; however, in the case of toxic oil syndrome, the symptoms were not always alleviated, and the treatment also did not prevent the disease from entering the chronic phase and, in the case of eosinophilia-myalgia syndrome, reduced muscle symptoms but unfortunately they recurred in the case of drug withdrawal. Moreover, treatment did not protect against transition to the chronic phase. Furthermore, it was observed that the HLA-DR4 haplotype predisposed patients to the chronic course of the disease, and in the case of toxic oil syndrome, the same form of the disease was observed for the HLA DR3 haplotype [13, 29].

Currently, these syndromes have only historical importance in the literature; however, descriptions of entities characterized by a similar clinical course and eosinophilia still appear and are associated with the consumption of various products. For example, Mendoza et al. reported two individual cases, for whom the symptoms known from a previously described syndrome occurred after consumption of a dietary supplement containing a probiotic, whose origin was unclear [30]. The condition of the patients improved after administration of glucocorticoids, although in both cases neurological deficits remained. This paper was not uncontroversial. Ouwehand et al. questioned the theory of Mendoza and ruled out a cause-effect relationship between probiotics and the observed symptoms. They supported this, inter alia, by pointing out the time which passed between the adoption of a dietary supplement (2-4 weeks) and the occurrence of symptoms. Indeed, probiotics remain in the gastrointestinal tract for approximately 1-2 weeks after consumption [31]. They also criticized the fact that in Mendoza's study an infectious agent which could also lead to eosinophilia was not excluded.

However, one should always remain vigilant and remember that sometimes dietary components may also be responsible for the occurrence of a disease.

\section{Summary}

Any abnormal laboratory or clinical picture of rheumatic disease, as well as cases which do not respond to conventional therapy, should be considered as possibly induced by drugs or environmental factors. It is so important because the main course of action is to eliminate the causative factor.

The authors declare no conflict of interest.

\section{References}

1. Xiao X, Chang C. Diagnosis and classification of drug-induced autoimmunity (DIA). J Autoimmun 2014; 48-49: 66-72.

2. Pretel M, Marquès L, España A. Drug-induced lupus erythematosus. Actas Dermosifiliogr 2014; 105: 18-30.

3. Lieberman MR, Liebman TN, Alapati U, Khachemoune A. TNF-inhibitor induced lupus in a patient treated with adalimumab for rheumatoid arthritis. Dermatol Online J 2014; 21(2). pii: 13030/qt18r2916d.

4. Quaresma MV, Bernardes Filho F, Oliveira FB, et al. Anti-TNF- $\alpha$ and hydralazine drug-induced lupus. An Bras Dermatol 2015; 90 (3 Suppl 1): 125-129.

5. Bukhari M. Drug-induced rheumatic diseases: a review of published case reports from the last two years. Curr Opin Rheumatol 2012; 24: 182-186.

6. Araújo-Fernández S, Ahijón-Lana M, Isenberg DA. Drug-induced lupus: Including anti-tumour necrosis factor and interferon induced. Lupus 2014; 23: 545-553.

7. Bernier MO, Mikaeloff $\mathrm{Y}$, Hudson M, Suissa S. Combined oral contraceptive use and the risk of systemic lupus erythematosus. Arthritis Rheum 2009; 61: 476-481.

8. Bove R. Autoimmune diseases and reproductive aging. Clin Immunol 2013; 149: 251-264.

9. Li RH, Gebbie AE, Wong RW, et al. The use of sex hormones in women with rheumatological diseases. Hong Kong Med J 2011; 17: 487-491.

10. Marzano AV, Tavecchio S, Menicanti C, Crosti C. Drug-induced lupus erythematosus. G Ital Dermatol Venereol 2014; 149: 301-309.

11. Harnett DT, Chandra-Sekhar HB, Hamilton SF. Drug-induced lupus erythematosus presenting with cardiac tamponade: a case report and literature review. Can J Cardiol 2014; 30: 247.e11-2.

12. Stöllberger C, Krutisch G, Finsterer J, Wolf HM. Dabigatran-induced lupus temporarily preventing blood group determination. Blood Coagul Fibrinolysis 2014; 25: 625-627.

13. D'Cruz D. Autoimmune diseases associated with drugs, chemicals and environmental factors. Toxicol Lett 2000; 112-113: 421-432.

14. Sánchez-Guerrero J, Karlson EW, Colditz GA, et al. Hair dye use and the risk of developing systemic lupus erythematosus. Arthritis Rheum 1996; 39: 657-662.

15. Zandman-Goddard G, Solomon M, Rosman Z, et al. Environment and lupus-related diseases. Lupus 2012; 21: 241-250.

16. Nelson P, Rylance P, Roden D, et al. Viruses as potential pathogenic agents in systemic lupus erythematosus. Lupus 2014; 23: 596-605.

17. Gianchecchi E, Fierabracci A. Gene/environment interactions in the pathogenesis of autoimmunity: new insights on the role of Toll-like receptors. Autoimmun Rev 2015; 14: 971-983.

18. Sharma SK, Handa R, Sood R, et al. Bleomycin-induced scleroderma. J Assoc Physicians India 2004; 52: 76-77.

19. Rünger TM, Adami S, Benhamou CL, et al. Morphea-like skin reactions in patients treated with the cathepsin $\mathrm{K}$ inhibitor balicatib. J Am Acad Dermatol 2012; 66: e89-96.

20. McCormic ZD, Khuder SS, Aryal BK, et al. Occupational silica exposure as a risk factor for scleroderma: a meta-analysis. Int Arch Occup Environ Health 2010; 83: 763-769.

21. Al Aranji G, White D, Solanki K. Scleroderma renal crisis following silicone breast implant rupture: a case report and review of the literature. Clin Exp Rheumatol 2014; 32: 262-266. 
22. Wilson RH, McCormick WE, Tatum CF, et al. Occupational acro-osteolysis. J Am Med Assoc 1967; 201: 577-581.

23. Ward AM, Udnoon S, Watkins J, et al. Immunological mechanisms in the pathogenesis of vinyl chloride disease. Br Med J 1976; 1: 936-938.

24. Straniero NR, Furst DE. Environmentally-induced systemic sclerosis-like illness. Baillieres Clin Rheumatol 1989; 3: 63-79.

25. Marie I, Gehanno JF, Bubenheim M, et al. Prospective study to evaluate the association between systemic sclerosis and occupational exposure and review of the literature. Autoimmun Rev 2014; 13: 151-156.

26. Marie I, Gehanno JF. Environmental risk factors of systemic sclerosis. Semin Immunopathol 2015; 37: 463-473.

27. Yamakage A, Ishikawa H, Saito Y, Hattori A. Occupational scleroderma-like disorder occurring in men engaged in the polymerization of epoxy resins. Dermatologica 1980; 161: 33-44.

28. Bovenzi M, Barbone F, Pisa FE, et al. Scleroderma and occupational exposure to hand-transmitted vibration. Int Arch Occup Environ Health 2001; 74: 579-582.

29. Philen RM, Posada M. Toxic oil syndrome and eosinophilia-myalgia syndrome: May 8-10, 1991, World Health Organization meeting report. Semin Arthritis Rheum 1993; 23: 104-124.

30. Mendoza FA, Purohit S, Kenyon L, Jimenez SA. Severe eosinophilic syndrome associated with the use of probiotic supplements: a new entity? Case Rep Rheumatol 2012; 2012: 934324.

31. Ouwehand AC, Rijkers GT. Severe eosinophilic syndrome: highly unlikely associated with the use of probiotic supplements! Case Rep Rheumatol 2013; 2013: 769127. 